\title{
Selección de materiales para la fabricación de hormigones de alta resistencia
}

\author{
M PILAR ALAEJOS GUTIÉRREZ \\ Dr. Ingeniero de Caminos, Canales y Puertos \\ División de Tecnología de Hormigones \\ Laboratorio Central de Estr. y Mat. (CEDEX) \\ MANUEL FERNÁNDEZ CÁNOVAS \\ Dr. Ingeniero de Construcción \\ Cated rático de Materiales \\ E.T.S.I. Caminos, Canales y Puertos (U.P.M.)
}

ESPAÑA

\begin{abstract}
RESUMEN
La selección previa de materiales constituyentes es el primer paso para conseguir un hormigón de calidad. La normativa española EH-91 establece diferentes requisitos que deben cumplir estos materiales para la fabricación de hormigones normales. Sin embargo, estos requisitos pueden no ser suficientes si se trata de fabricar un hormigón de alta resistencia.

En el artículo se muestran ensayos realizados con hormigones de alta resistencia, que han permitido establecer una sistemática de selección de materiales constituyentes para estos hormigones: Cementos,áridos y aditivos. Los resultados permiten, mediante ensayos habituales de Laboratorio, conocer la potencial aptitud de estos materiales para la fabricación de hormigones de alta resistencia. Las exigencias deben ser mayores cuanto mayores sean las resistencias que se quieran alcanzar. La metodología expuesta permite ahorrar muchos ensayos previos de Laboratorio que hasta el momento deben realizarse, cuando se quiere conseguir un hormigón de estas características.
\end{abstract}

\section{SUMMARY}

The selection of component materials is the first step in order to reach a good quality concrete. The Spanish Code EII-9I point out the different requisites for the component materials used in a normal concrete. However, these requisites may be not sufficient for a high strength concrete (HSC).

In this paper we present the tests carried out in order to stablish a system for selecting the component materials for a HSC, such us: cements, aggregates, admixtures. The results of the tests allow by means of normal laboratory tests to know the suitability of these materials used for making HCS. The requeriments for these component materials must be higher when higher are the strengths to be reached. The exposed method allow us to save a lot of previous laboratory tests neccesary until now, in order to get a high strength concrete.

\section{1.- INTRODUCCIÓN}

La selección de los materiales constituyentes es el primer paso necesario para la fabricación de un hormigón. En la tecnología del hormigón normal a esta selección no se le suele prestar una excesiva atención, lo cual es origen de problemas en numerosas ocasiones. Para la fabricación de hormigón de alta resistencia la selección previa de materiales se puede considerar crítica si se desea tener garantías de éxito, ya que se trata de obtener un hormigón de elevada calidad y de elevadas prestaciones.
En el caso del hormigón convencional, la propia EH-91 contempla diversos requisitos que deben cumplir el cemento, el agua y los áridos, que son los elementos básicos de un hormigón normal, así como algunas consideraciones sobre aditivos químicos ociertas adiciones minerales, hoy en día ampliamente utilizadas. Requisitos adicionales al cemento se recogen en la Instrucción de cementos RC-93. Todas las exigencias impuestas están encaminadas a la obtención de hormigones con las características deseadas y una durabilidad adecuada.

Sin embargo, la EH-91 tiene un campo de validez que 
comienza con el hormigón de categoría $\mathrm{H}-125$ y termina en el de categoría H-500, límite en el que precisamente nacen los hormigones de alta resistencia (HAR). Por supuesto, toda la formulación empírica, métodos de cálculo e incluso detalles de ejecución recogidos en la EH-91, están avalados por la amplia experiencia que ha proporcionado la utilización de hormigones normales desde hace mucho tiempo. Sin embargo, una gran parte de los aspectos contemplados en la Instrucción española, como sucede con otras normativas internacionales, puede no ser válida para los hormigones de alta resistencia. Estos hormigones presentan unas propiedades y características propias, que no son una mera extrapolación de las que tiene el hormigón normal.

Comenzando por la tecnología básica de fabricación de un hormigón de alta resistencia, los requisitos exigibles a los materiales que forman las mezclas de hormigón normal pueden noser suficientes si se quiere conseguir un hormigón de elevadas prestaciones.

La bibliografía recoge características de tipogeneral sobre los requisitos a exigir a los materiales componentes de un hormigón de alta resistencia: Aridogrueso de machaqueo, de buena calidad y de 12-14 mm de tamaño máximo, arena rodada, cementos de elevada categoría resistente, superplastificantes compatibles con el cemento elegido, etc. Estas indicaciones de carácter general no resultan suficientes, ya que la experiencia demuestra que antes de la realización de una aplicación práctica con este hormigón, los estudios previos de Laboratorio para la selección de los materiales constituyentes deben ser muy amplios, ya que no existen exigencias concretas avaladas por suficiente experimentación.

En el Laboratorio Central de Estructuras y Materiales del CEDEX se ha desarrollado un programa experimental sobre hormigones de alta resistencia. Uno de los objetivos principales ha sido la realización de una guía de preselección de materiales para fabricar hormigón de alta resistencia, partiendo como base de las recomendaciones generales encontradas en la bibliografía, y añadiendo otras más concretas procedentes de la experimentación propia. Estaguía de preselección de materiales, aunque no permitirá suprimir totalmente los ensayos previos de Laboratorio sobre materiales potencialmente aptos para HAR, sí permitirá partir de una orientación inicial que puede ahorrar mucho trabajo.

\section{2.- SELECCIÓN DE MATERIALES}

La selección de materiales para un hormigón de alta resistencia debe partir de dos objetivos fundamentales:

-Conseguir las máximas resistencias: Aunque este puede parecer un objetivo obvio, hay que tener en cuenta que se pueden llegar a fabricar hormigones de alta resistencia, por ejemplo, con cantidades muy altas de humo de sílice, o relaciones agua/cemento extremadamente bajas y por tanto con grandes contenidos de aditivo superplastificante. Pero también es posible obtener la misma resistencia en condiciones menos extremas y centrándose en una optimización de los componentes de la mezcla. Este, por tanto, es un objetivo de partida: Obteniendo el máximo rendimiento de todos los componentes de la mezcla se conseguirá la máxima resistencia al mínimo coste.

-Conseguir consistencias adecuadas: Si este es un objetivo importante en la tecnología del hormigón normal, resulta primordial cuando se trata de fabricar hormigón de alta resistencia. Las relaciones agua/cemento tan bajas a las que es necesario llegar para conseguir determinados niveles de resistencia, obliga a que en la selección de materiales se dirija fundamentalmente a elegir a aquellos que demanden la mínima cantidad posible de agua.

Por tanto, los ensayos para evaluar la potencial utilización de un material para fabricar hormigón de alta resistencia se deben centrar en que proporcione la máxima resistencia con la mínima demanda de agua. En aquellos materiales en cuya selección ambas exigencias se traducen en efectos contrapuestos, la selección se debe basar en aquel efecto que sea preponderante.

Con estas premisas, se desarrolló un plan de ensayos para determinar los requisitos exigibles a los distintos componentes presentes en las mezclas de hormigón de alta resistencia.

\section{1.- Cemento}

Parece lógico pensar que para fabricar un hormigón de alta resistencia sea necesario utilizar cementos comerciales de la máxima categoría en cuanto a resistencias mecánicas se refiere, es decir, en el caso español el Tipo I55. El escoger a priori cementos Tipo I, con un máximo permitido del $5 \%$ de adiciones, proviene del hecho de que el propio HAR llevará en numerosas ocasiones adición de humo de sílice, que necesita clínker puro hidratado para reaccionar puzolánicamente e incrementar las resistencias, por lo que no conviene la utilización de cementos con adiciones.

Se hicieron ensayos para evaluar el incremento de resistencia que proporciona un cemento Tipo I55 frente a uno de categoría inferior Tipo I45. Se utilizaron cementos de alta resistencia inicial, ya que desde un punto de vista práctico interesa facilitar reacciones a corto plazo, ya que cualquier pérdida de humedad a edad temprana ralentiza todas las reacciones, de hidratación y puzolánicas. En general cementos de reacción más lenta exigen curados más prolongados en el tiempo que cementos de alta resistencia inicial, cuyo curado debe cuidarse mucho, pero a edades tempranas. En este sentido, hay que apuntar que 
el HAR desarrolla en general resistencias muy rápidamente, debido a las bajas relaciones agua/cemento empleadas, 10 cual aproxima físicamente a las partículas reactivas (cemento y humo de sílice), densificando muy rápidamente su estructura sólida.

Algunas de las características de los dos cementos utilizados se muestran en el Cuadro 1. La resistencia del mortero normalizado del cemento I55-A se puede cifrar en un 15\% por encima de la que presentó el I45-A. Ello es debido fundamentalmente a la mayor superficie específica que presenta el de mayor categoría, como se observa en el cuadro. Asimismo, es interesante destacar la influencia que la mayor superficie específica produce en el tiempo de fraguado. El I55-A reacciona más rápidamente, comienza a fraguar antes y finaliza el fraguado también más rápidamente.

En la Fig.1 se observan los resultados de resistencia con ambos tipos de cemento y distintos porcentajes de humo de sílice $(0 \%, 10 \%$ y $15 \%)$, en el rango de relación agua/ cemento de 0,26 a 0,40 . En la figura se han dibujado las regresiones correspondientes a los tres porcentajes para el cemento I45A. El incremento de resistencia conseguido con el I55A es muy leve, de 30 a $50 \mathrm{kp} / \mathrm{cm}^{2}$ en todo el rango de relaciones agua/cemento ensayado.

El hecho de que el incremento de resistencia observado en el mortero normalizado no se reproduzca en el hormigón puede estar relacionado con la relación agua/cemento utilizada en ambos casos. En el mortero se utiliza a/c igual a 0.5. En un cemento de alta superficie específica (como el Tipo I55-A utilizado), una situación de elevado contenido de agua hace que se hidrate una gran cantidad de cemento consiguiéndose un incremento de resistencia cifrado en el $15 \%$. Conforme se reduce la relación a/c la reacción rápida del I55-A consume una gran parte del agua de amasado, y se estabilizan pronto las reacciones puzolánicas y de hidratación, quedando probablemente una parte del cemento sin hidratar por falta de agua (la red de productos de hidratación formada es tan densa que incluso hace difícil el aporte de agua desde el exterior). El I45-A reacciona más lentamente por su menor superficie especifica, pero continúa ganando resistencia hasta consumir gran parte del agua de amasado, momento en el cual casi ha alcanzado las resistencias que ha conseguido el I55-A más rápidamente.

Posiblemente pudiera aprovecharse el incremento de resistencia que proporcionan los cementos I55-A si éstos basaran éste en una composición química mejor ajustada, en vez de una mayor finura de molido.

Aunque las resistencias obtenidas con el I55-A no fueron sensiblemente mayores a las del I45-A, lo que sí se observó claramente es que este cemento produjo una disminución notable de consistencia, tal como se muestra en las Figs. 2a y $2 b$. Todas las masas fabricadas con el cemento I45A tuvieron asientos y mesas muy superiores a las mismas masas fabricadas con el cemento I55-A.

Lógicamente, la mayor finura que presentan en general los cementos I55-A disminuye la efectividad de los superplastificantes, ya que éstos actúan sobre la superficie de las partículas de cemento, mucho mayor en estos cementos. Por ello será necesario mayor contenido de aditivo, para cubrir el exceso de superficie específica y separar convenientemente las partículas. Las peores consistencias registradas también pudieron influir en una disminución de resistencia debido a una posible peor compactación de las probetas. En cualquier caso los ensayos realizados con un cemento I55-A no aportaron incrementos sensibles de resistencia y sin embargo si empeoraron notablemente la consistencia.

Teniendo en cuenta los dos objetivos fundamentales para la selección de materiales para hormigón de alta resistencia, máxima resistencia pero mínima demanda de agua, en el caso del cemento puede decantarse la selección por cementos Tipo I45-A, que producirán unaleve disminución de resistencia, que sin embargo pueden compensar con creces permitiendo llegar sin problemas a relaciones agua/ cemento más bajas.

Otro factor importante a la hora de seleccionar el cemento es el de utilizar cementos con tiempo de fraguado más rápido, sobre todo si se van a emplear contenidos de aditivo muy elevados (por encima del $3 \%$ ). Ello es debido al efecto de retraso de fraguado que el superplastificante, en grandes cantidades, produce sobre el cemento. Este retraso se acusa antes en cementos con fraguado más lento. En concreto, y teniendo en cuenta los datos de fraguado que aparecen en el Cuadro 1 , el cemento I45-A no admitía más de un $3 \%$ de aditivo, ya que si se superaba este límite se producían importantes retrasos de fraguado: A las 24 horas las probetas no podían ser desmoldeadas. Con el cemento I55-A se fabricaron amasadas hasta con un 3.5\% de aditivo sin problemas de este tipo, que probablemente se producirán con un límite superior, al ser un cemento que inherentemente fragua más rápido.

Por tanto, y como resumen de los requisitos exigibles al cemento podemos citar:

.Es mejor seleccionar cementos con máximas resistencias conseguidas con una composición química ajustada y no con mayor superficie específica.

.Si no es posible cumplir el punto anterior, es mejor seleccionar cementos de Tipo I45-A, en general con una superficie específica no muy elevada, que cementos Tipo I55-A, en general con elevada finura de molido. La demanda de agua de estos últimos no compensa el incremento de resistencia conseguido, muy inferior al que 
presentan en mortero normalizado, o en hormigón normal.

.Una vez cumplidas las consideraciones anteriores, es aconsejable que los tiempos de fraguado del cemento (controlados mediante ensayo UNE 80-102) no sean muy dilatados. Se pueden producir problemas de retrasos con altas cantidades de superplastificante en cementos con un principio de fraguado superior a las 3 horas.

\section{CUADRO 1}

\begin{tabular}{|c|c|c|c|c|c|c|}
\hline $\begin{array}{c}\text { CEMENTO } \\
\text { TIPO }\end{array}$ & $\begin{array}{c}\mathrm{Al}_{2} \mathrm{O}_{3} \\
(\%)\end{array}$ & $\begin{array}{c}\mathrm{Fe}_{2} \mathrm{O}_{3} \\
(\%)\end{array}$ & $\begin{array}{c}\text { BLAINE } \\
\left(\mathrm{cm}^{2} / g r\right)\end{array}$ & $\begin{array}{c}f_{c, 28 d} \\
M P a\end{array}$ & P.FRAGUADO & F.FRAGUADO \\
\hline \hline I-45A & 5,26 & 5,12 & 3579 & 50,8 & $3 \mathrm{~h} 15 \mathrm{~m}$ & $4 \mathrm{~h} 30 \mathrm{~m}$ \\
\hline $\mathrm{I}-55 \mathrm{~A}$ & 5,49 & 2,42 & 4526 & 58,2 & $2 \mathrm{hOm}$ & $3 \mathrm{hOm}$ \\
\hline
\end{tabular}

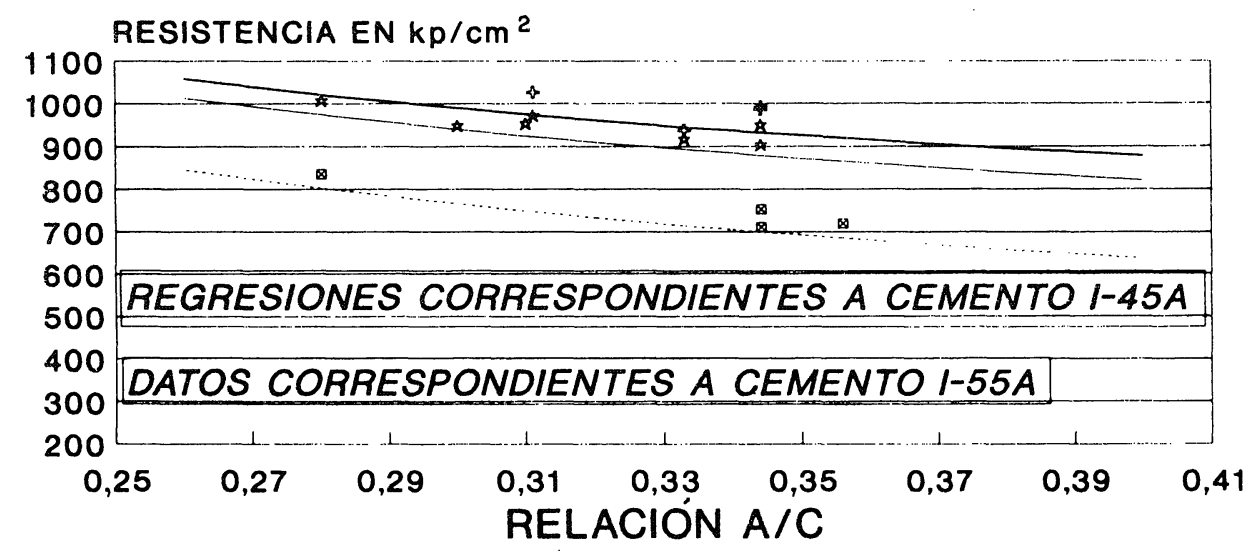

\begin{tabular}{lllll}
\hline 10\%MS 155A & $*$ 15\%MS 155A & $\cdots$ & REG.10\%MS 145A \\
- REG.15\%MS 145A $\$$ O\%MS 155A & $\cdots$ & REG.0\%MS 145A
\end{tabular}

Fig. 1.- Comparación de resistencias (cemento 145 A y 155 A).

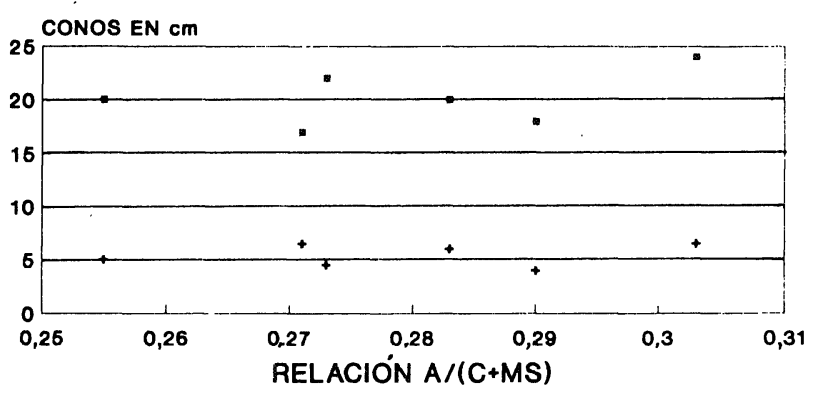

- CEMENTO 145A + CEMENTO I55A

Fig. 2a.- Consistencias 145A y 155A (cono de Abrams).

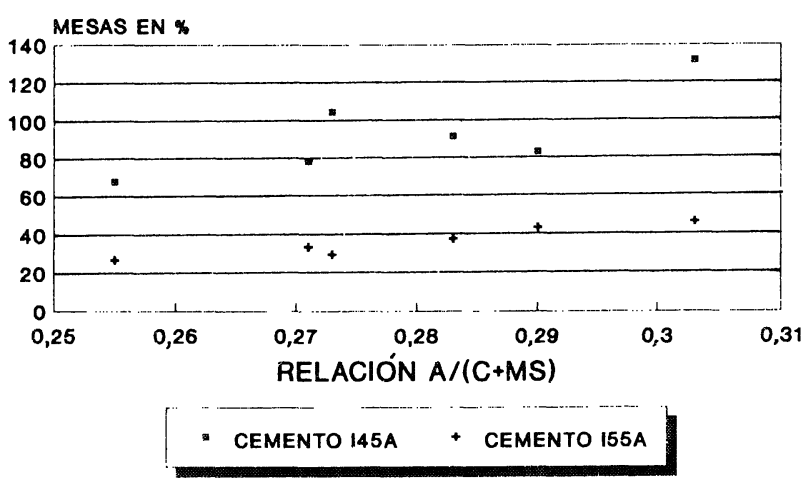

Fig. 2b.- Consistencias 145A y 155A (mesa de sacudidas). 


\section{2.- Superplastificante}

La compatibilidad entre el cemento y superplastificante elegidos es el punto fundamental para alcanzar determinados niveles de resistencia. Para relaciones agua/ cemento extremadamente bajas, la efectividad del aditivo sobre el cemento debe ser máxima, si no se quiere tener problemas de consistencia.

Se puede llegar a pensar que este problema de mayor o menor efectividad del aditivo se puede solventar añadiendo a la mezcla menores o mayores cantidades de superplastificante. Efectivamente, mayores cantidades de aditivo mejoran la consistencia, como se observa en la Fig. 3 ( $a$ y b), donde un incremento de $0,75 \%$ de aditivo convirtió un hormigón de consistencia seca en consistencia fluida. Sin embargo no se puede añadir excesivas cantidades de aditivo, existiendo un límite (que depende del propio cemento) en el que comienzan a presentarse problemas de fraguado, como ya ha sido comentado con anterioridad.

Por tanto, resulta esencial con relaciones agua/cemento muy bajas prestarle la debida atención a la selección conjunta cemento-aditivo.

El mejor o peor comportamiento de un aditivo superplastificante con un determinado cemento, es un fenómeno que en parte es aun desconocido. Las investigaciones parecen apuntar a que el contenido de alúmina del cemento juega un papel importante en esta compatibilidad. Sin embargo, aunque se limite el contenido de alúmina del cemento se pueden presentar problemas de consistencia. De hecho, tal como se observa en el Cuadro 1, los dos cementos utilizados en las pruebas de selección de cemento presentaron un contenido de alúmina muy similar, alrededor del $5 \%$. Sin embargo, como ha quedado patente en el apartado anterior el comportamiento del hormigón con ellos fabricado, difirió mucho de uno a otro en cuanto a consistencia se refiere. Lógicamente, además de la composición química, la finura del cemento también juega un papel importante en el comportamiento del binomio cemento-superplastificante. Los resultados alcanzados hasta el momento en las distintas investigaciones, no permiten realizar la selección del binomio adecuado mediante ensayos previos de caracterización, y es necesario acudir a ensayos de comportamiento.

Desde luego, la mejor forma de conocer el comportamiento de un cementoy un superplastificante esfabricar hormigón con ambos y medir la consistencia. Sin embargo, este es un método algo engorroso si han de realizarse pruebas con varios cementos y superplastificantes.

Para evaluar esta compatibilidad se empleó el método basado en el cono de Marsh, que se realizó en pasta con cemento, microsílice, aguay aditivo. El ensayo consiste en llenar el cono con la pasta hasta enrasar a una marca realizada en el interior del embudo. El embudo permanece cerrado con un tapón durante la operación de llenado. Una vez terminada ésta se quita el tapón y simultáneamente se acciona un cronómetro. La pasta comienza a caer por el embudo y se mide el tiempo de caída continua de la misma. Esta operación se realizó cada media hora, advirtiéndose asíla pérdida de consistenciaen el tiempo. Si la consistencia de la pasta no es la adecuada para que la caída sea continua y ésta se realiza interrumpidamente, se da el ensayo por terminado. Este procedimiento de ensayo se basa en el descrito por el ASTM C939, aunque las dimensiones del embudo utilizado no se corresponden exactamente con las descritas en dicha norma. Los resultados permitían comparar la mayor o menor efectividad de distintos aditivos con diferentes cementos, en cuanto a mayor fluidez y constancia en el tiempo del efecto fluidificante.

La pasta de cemento se amasó con una relación agua/ cemento de 0,35 y se dosificaron cantidades variables de superplastificante, comprendidas entre el 2 y el $3 \%$. Todas las mezclas llevaban un $10 \%$ de microsílice, ya que la microsílice tiene una influencia importante en la trabajabilidad (demanda gran cantidad de agua) e iba a ser utilizada durante todoel plan de ensayos, así que interesaba conocer su influencia sobre la consistencia en esta fase de pruebas.

Los resultados utilizando únicamente este ensayo, sin embargo, no parecen definitivos, si se observa el Cuadro 2a, donde dos cementos Tipo I45-A pero de distintas cementeras (que hemos denominado Fuente A y Fuente B), dieron similares resultados en fluidez de la pasta medida mediante este ensayo, pero produjeron consistencias muy diferentes en hormigón, comose observa en el Cuadro $2 b$.

Lógicamente, la compatibilidad cemento-aditivo para producir consistencias adecuadas, depende en primer lugar de la propia demanda de agua que presenta el cemento. Ésta puede evaluarse mediante el ensayo de escurrimiento en la mesa de sacudidas para mortero normalizado, e incluso mediante la determinación del agua de consistencia normal (A.C.N.) de un cemento, según la norma UNE 80-101. Los resultados de estos ensayos, para cementos de diferente categoría

(I-45A y I-55A) y fuentes de procedencia distinta se muestran en el Cuadro 3, junto con los del ensayo basado en el del Cono de Marsh.

Se observa en este cuadro que un escurrimiento del mortero en la mesa de sacudidas alto, como en el caso del cemento "Número 1", es determinante a la hora de proporcionar asientos altos en el hormigón. Pero por otra parte, a igualdad en el valor de la mesa, un mejor comportamiento en el ensayo realizado basado en el Cono de Marsh, mejora las consistencias en el hormigón. El 
agua de consistencia normal, no parece tener mucha sensibilidad a la hora de determinar con exactitud la demanda de agua de un determinado cemento.

Por tanto, y de acuerdo a los resultados obtenidos, una forma de conocer a priori si el cemento funcionará bien con el superplastificante elegido es determinar el escurrimiento del mortero normalizado en la mesa de sacudidas:

.Si su valor es superior al $70 \%$ se trata de un cemento con una demanda de agua muy baja, que previsiblemente funcionará muy bien con relaciones agua/cemento bajas, y será fácil encontrar un aditivo que actúe bien con este cemento.

Si el resultado está entre el $60-70 \%$, es un cemento con una demanda de agua media, su funcionamiento en relaciones agua/cemento bajas dependerá del superplastificante empleado. Probando con diferentes superplastificantes, se puede encontrar el que mejor funcione con el cemento, utilizando el ensayo basado en el cono de Marsh. Según los resultados por nosotros obtenidos, la combinación aditivo-cemento será buena cuando se obtenga una fluidez con un $2 \%$ de aditivo. Probablemente la evaluación de lacompatibilidad también se pueda hacer mediante una proporción predeterminada cemento-aditivo en mortero, utilizando en este caso la mesa de sacudidas.

Los cementos con un escurrimiento inferior al $60 \%$ son cementos con alta demanda de agua. Será difícil encontrar un superplastificante que permita emplearlos en relaciones agua/cemento muy bajas.

Mediante la realización de estos ensayos, se puede llegar a conseguir una selección rápida de cemento-aditivo. Conviene utilizar la metodología en fase de preselección, descartando los binomios de peor funcionamiento. Así se pueden reducir al máximo las pruebas con hormigón, que siempre deben realizarse para la selección final del binomio óptimo.
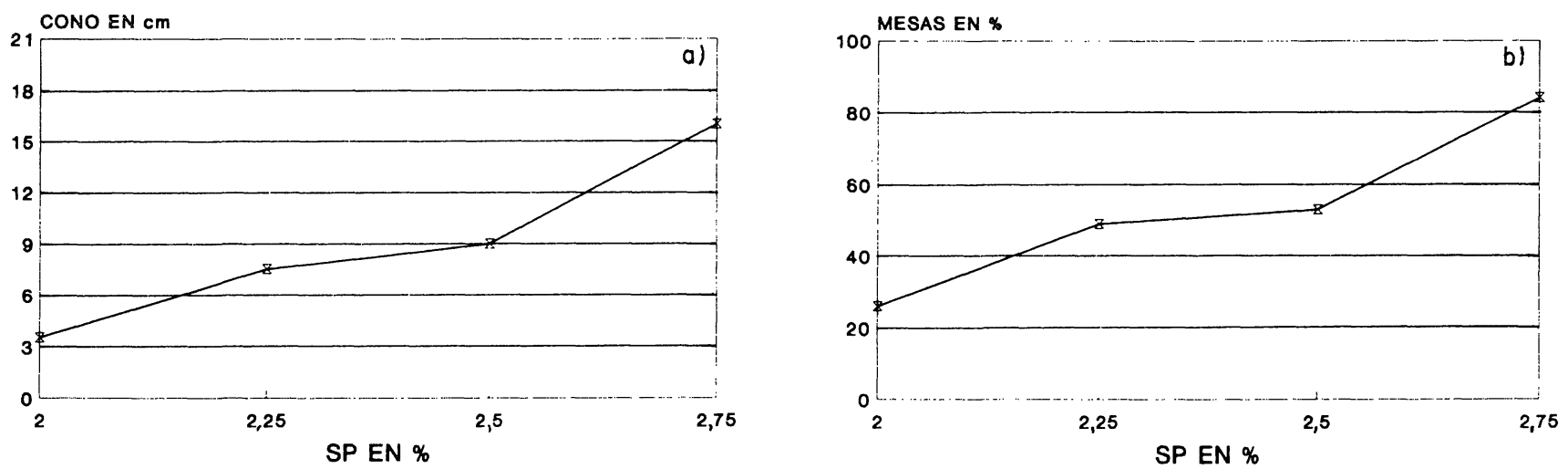

Fig.3 (a y b).- Influencia del superplastificante en la consistencia.

CUADRO $2 \mathrm{a}$

\begin{tabular}{|c|c|c||}
\hline \multicolumn{3}{|c|}{ CEMENTO TIPO I-45A } \\
\hline $\begin{array}{c}\text { TIEMPO } \\
\text { MEDIDA }\end{array}$ & $\begin{array}{c}\text { FUENTE A } \\
\text { aditivo d al 3\% }\end{array}$ & $\begin{array}{c}\text { FUENTE B } \\
\text { aditivo d al 3\% }\end{array}$ \\
\hline $5 \mathrm{~m}$ & $13 \mathrm{~s}$ & $23 \mathrm{~s}$ \\
\hline $30 \mathrm{~m}$ & $17 \mathrm{~s}$ & $23 \mathrm{~s}$ \\
\hline $60 \mathrm{~m}$ & $19 \mathrm{~s}$ & $22 \mathrm{~s}$ \\
\hline
\end{tabular}


CUADRO $2 b$

\begin{tabular}{|c|c|c|c|c|c|c|c||}
\hline MARCAS & $\begin{array}{c}\text { AGUA } \\
(\mathbf{l})\end{array}$ & $\begin{array}{c}\text { CEMENTO } \\
\left(\mathrm{kg} / \mathrm{m}^{3}\right)\end{array}$ & $\begin{array}{c}\text { FUENTE } \\
\text { CEMENTO }\end{array}$ & $\begin{array}{c}\text { SP } \\
(\%)\end{array}$ & $\begin{array}{c}\text { CONO } \\
(\mathrm{cm})\end{array}$ & $\begin{array}{c}\text { MESA } \\
(\%)\end{array}$ & $\begin{array}{c}\text { fc28d } \\
\left(\mathbf{k p} / \mathrm{cm}^{2}\right)\end{array}$ \\
\hline 26BRB & 160 & 400 & A & 3 & 4 & 39 & 634 \\
\hline 26BRA & 160 & 400 & B & 2 & 22 & 80 & 625 \\
\hline 19BR & 150 & 400 & A & 3 & 4 & 35 & 668 \\
\hline 19BRI & 150 & 400 & B & 2,5 & - & 83 & 671 \\
\hline
\end{tabular}

CUADRO 3

\begin{tabular}{|c|c|c|c|c|c|}
\hline $\begin{array}{c}\text { FUENTE } \\
\text { CEMENTO }\end{array}$ & A.C.N & $\begin{array}{c}\text { MESA } \\
\text { MORTERO }\end{array}$ & $\begin{array}{c}\text { CONO } \\
\text { MARSH }\end{array}$ & $\begin{array}{c}\text { CONO } \\
\text { ABRAMS }\end{array}$ & MESA \\
\hline NÚMERO 1 & 0,29 & $76 \%$ & $\begin{array}{c}\text { Os }(2 \%) \\
\text { Os }(2.5 \%) \\
23 s \quad(3 \%)\end{array}$ & $20 \mathrm{~cm}$ & $123 \%$ \\
\hline NÚMERO 2 & 0,27 & $61 \%$ & $19 \mathrm{~s}(2 \%)$ & $19 \mathrm{~cm}$ & $95 \%$ \\
\hline NÚMERO 3 & 0,305 & $55 \%$ & Os $(3 \%)$ & $6 \mathrm{~cm}$ & $56 \%$ \\
\hline NÚMERO 4 & 0,28 & $70 \%$ & $\begin{array}{c}\text { Os }(2 \%) \\
\text { Os }(2.5 \%) \\
8 s(3 \%)\end{array}$ & $10 \mathrm{~cm}$ & $62 \%$ \\
\hline NÚMERO 5 & 0,295 & $72 \%$ & $\begin{array}{l}\text { Os }(2 \%) \\
11 s(2.5 \%) \\
10 s(3 \%)\end{array}$ & $18 \mathrm{~cm}$ & $84 \%$ \\
\hline NÚMERO 6 & 0,29 & $68 \%$ & $\begin{array}{c}\text { Os }(2 \%) \\
\text { Os }(2.5 \%) \\
20 s(3 \%)\end{array}$ & $4 \mathrm{~cm}$ & $44 \%$ \\
\hline
\end{tabular}

\section{3.- Microsilice}

El humo de sílice es la puzolana artificial más activa de las comercializadas en la actualidad. Ello es debido a que presenta dos características fundamentales: Un contenido muy elevado de dióxido de silicio en estado amorfo y una extremada finura.

Los fabricantes de aditivos comercializan normalmente el humo de sílice premezclándolo con un aditivo superplastificante en polvo, a fin de compensar la gran demanda de agua que produce en el hormigón esta adición. Sin embargo, en el plan de ensayos se decidió utilizar el humo de sílice puro, sin aditivo químico, para no introducir nuevas variables y poder controlar perfectamente el contenido y tipo de superfluidificante que se iba a utilizar.

Existe en la actualidad una normaUNE 83-460 que recoge las especificaciones que debe cumplir el humo de sílice para utilizarlo como adición al hormigón. Incluso cumpliendo estas especificaciones se puede elegir humo de sílice más o menos activo según su composición química y finura.

Se eligieron muestras de dos casas comerciales que suministraron el humo de sílice puro, sin aditivos superfluidificantes. Se realizó una determinación del contenido de dióxido de silicio de ambas según norma UNE 80-215-88. Los resultados fueron de $80.3 \%$ y $96.8 \%$ respectivamente. En la Fig. 4 se observa la influencia del contenido de $\mathrm{SiO}_{2}$, de la microsílice sobre la resistencia de probetas de mortero. El gráfico se ha obtenido a través de los resultados de un estudio realizado en el Laboratorio de Ponts et Chausees. Como se ve en la figura, las microsílices más puras proporcionan algo más de resistencia, por su mayor actividad puzolánica. Por ello se decidió utilizar la que mayor contenido de dióxido de silicio presentaba.

El humo de sílice es un polvo de color gris oscuro, tal como se aprecia en la Fotografía 1, en comparación con el 
cemento. Esta adición tiñe de color gris oscuroel hormigón, tal como se aprecia en la Fotografia 2, en la cual la probeta de la derechatiene unacantidad determinadade microsílice y la de la izquierda no lleva adición. Este es un dato a tener en cuenta si el hormigón va a quedar visto.

Una característica fundamental del humo de sílice es su extremadafinura, que lo hace muy reactivo conel hidróxido cálcico desprendido en las reacciones de hidratación del cemento. En la Fotografía 3 se aprecia el aspecto del humo de sílice a través del microscopio electrónico a 20000 aumentos. Asimismo se aprecia la perfecta esfericidad de las partículas de humo de sílice. Como comparación se muestra la Fotografía 4 en la que se ven las partículas de cemento tipo I45-A utilizado en el plan de ensayos, a 2.000 aumentos. Estas fotografias fueron tomadas por la División de Mineralogía y Petrología.
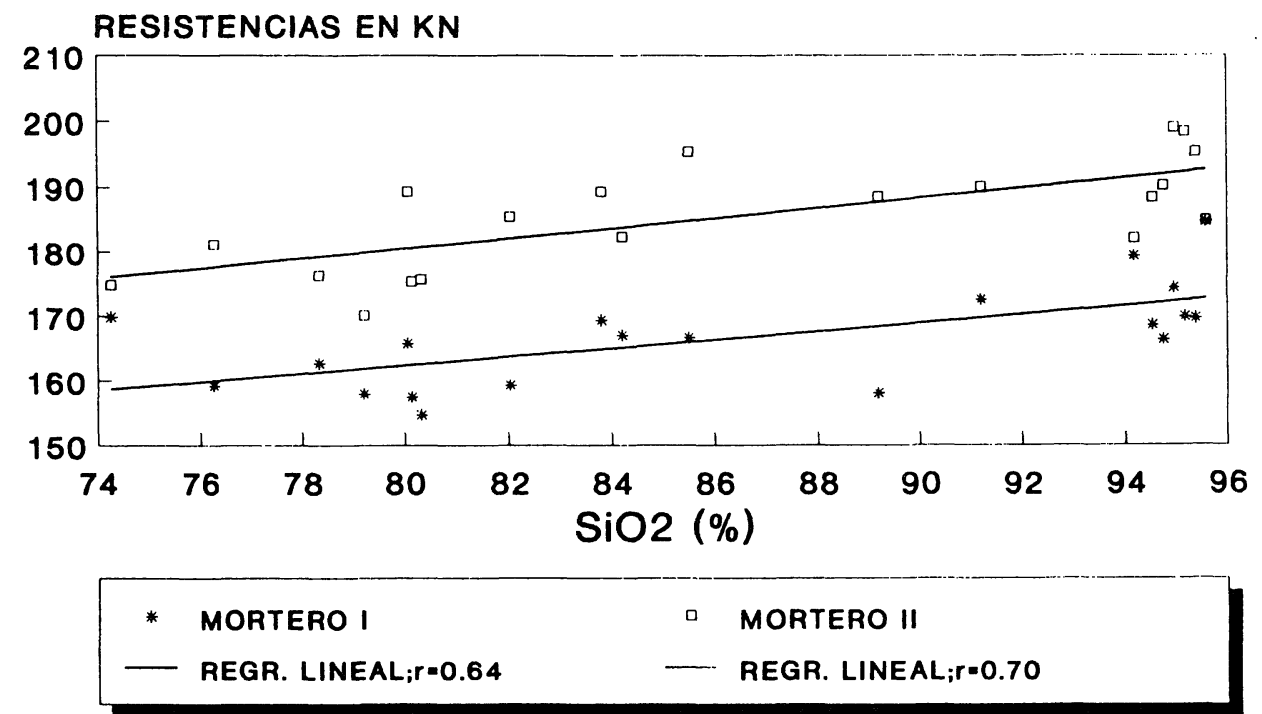

Fig.4.- Influencia de la MS en la resistencia (referencia Larrard et al, 1990)

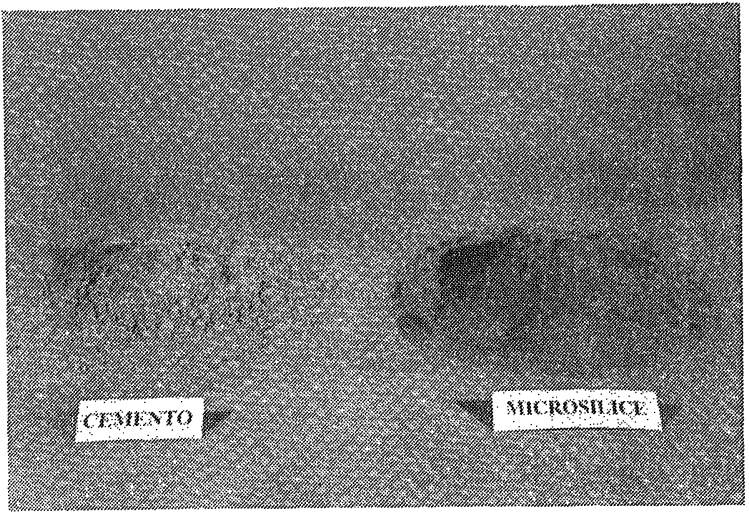

Foto 1

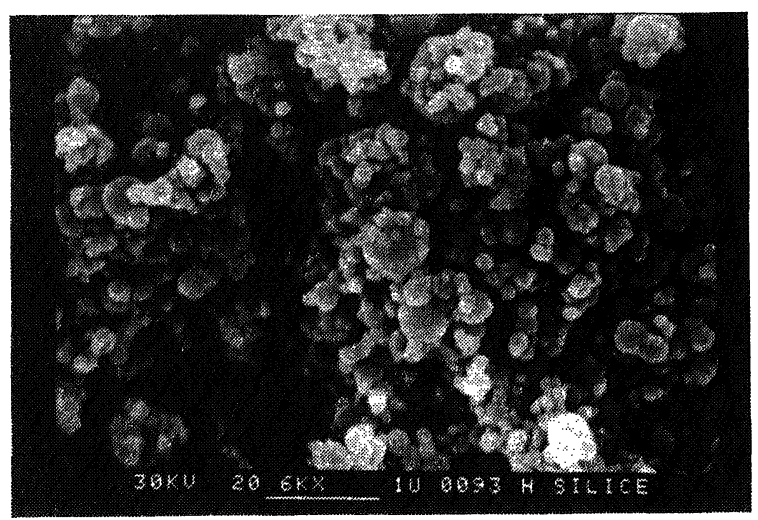

Foto 3

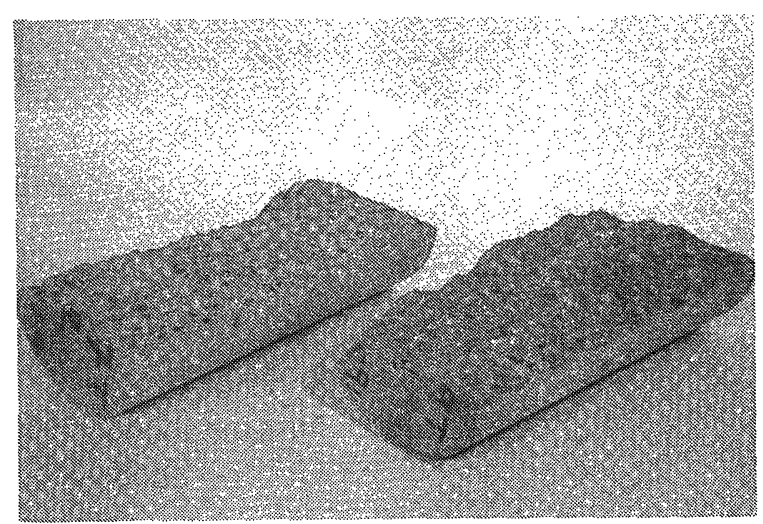

Foto 2

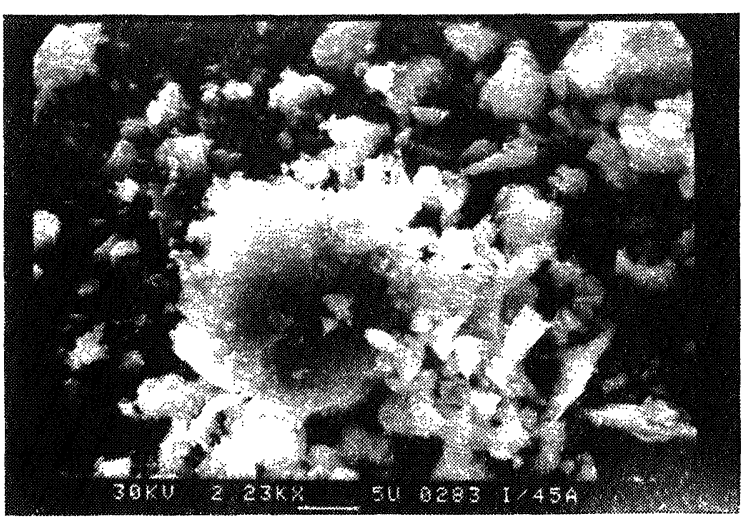

Foto 4 


\section{4.- Áridos}

La arena a utilizar en mezclas de hormigón de alta resistencia viene determinada por la necesidad de que demande la mínima cantidad de agua. Ello restringe la utilización a arenas rodadas, que suelen tener un origen silícico cuarcítico, tratándose en general de arenas muy resistentes.

En cuanto al árido grueso, la bibliografía especializada señala la importancia de la selección cuidada del árido para fabricar hormigón de alta resistencia. Las propiedades del árido influyen a su vez en las propiedades del hormigón fabricado con él, fundamentalmente en la consistencia del hormigón fresco y en las propiedades mecánicas del hormigón endurecido. Factores a tener en cuenta para seleccionar un árido para hormigón de alta resistencia son una elevada resistencia, buen coeficiente de forma y adecuadagranulometría. Sin embargo, noexisten requisitos concretos para descartar o elegir un árido determinado.
En el plan experimental llevado a cabo, se utilizaron varios áridos procedentes de diferentes puntos de España, para estudiar la influencia de las propiedades del árido sobre la consistencia y resistencia del hormigón.

En el Cuadro 4 se muestran los resultados de los ensayos realizados sobre los distintos áridos utilizados. En el Cuadro 5 los resultados de consistencia y resistencia para la misma dosificación con áridos diferentes. Las dosificaciones marcadas "FXT12P" y "HSC18P" son la misma, salvo la partida de superplastificante utilizada. Por este motivo existen diferencias en la consistencia medida e incluso en las resistencias obtenidas, ya que la primera partida producía conos inferiores y menores resistencias. La importancia de controlar las partidas de materiales, incluido el aditivo fue también objeto de estudio duranteel programade investigación. Los resultados de esta fase se recogerán en una próxima publicación. Se repitió en el caso de la caliza la misma dosificación con ambas partidas de aditivo, lo cual permite un nexo de unión comparativo entre los dos grupos de dosificaciones.

CUADRO 4

\begin{tabular}{|c|c|c|c|c|c|c||}
\hline ÁRIDO & $\begin{array}{c}\text { DENSIDAD } \\
\left(\mathrm{T} / \mathrm{m}^{3}\right)\end{array}$ & $\begin{array}{c}\text { ABSORCIÓN } \\
(\%)\end{array}$ & C.FORMA & $\begin{array}{c}\text { D MÁXIMO } \\
(\mathrm{mm})\end{array}$ & LOS ANGELES & I.MACHAC. \\
\hline ESQUISTO & 2,65 & 0,97 & 0,20 & 12 & 13 & 12 \\
\hline PÓRFIDO & 2,67 & 0,96 & 0,21 & 14 & 17 & 12 \\
\hline MONZONITA & 2,59 & 1,05 & 0,26 & 12 & 13 & 11 \\
\hline BASALTO 1 & 2,90 & 2,29 & 0,26 & 11 & 13 & 13 \\
\hline CALIZA & 2,50 & 2,44 & 0,27 & 10 & 26 & 27 \\
\hline BASALTO 2 & 2,99 & 1,20 & 0,21 & 12 & 15 & 9 \\
\hline OFITA & 2,54 & 3,46 & 0,24 & 13 & 23 & 17 \\
\hline
\end{tabular}

CUADRO 5

\begin{tabular}{|c|c|c|c|c|c||}
\hline \multicolumn{7}{|c|}{ AMASADAS CON 450 KG DE CEMENTO, 15\% DE MS Y 150 I DE AGUA } \\
\hline ÁRIDO & MARCAS & PARTIDA SP & CONO & MESA & RESISTENCIA \\
\hline \hline PÓRFIDO & FXT12P & PRIMERA & 18 & 84 & 942 \\
\hline CALIZA & CALIZA4 & PRIMERA & 9 & 56 & 1042 \\
\hline BASALTO 1 & BASALTO 1 & PRIMERA & 3 & 32 & 1087 \\
\hline \hline PÓRFIDO & HSC18P & SEGUNDA & 21 & 131 & 994 \\
\hline MONZONITA & MONZONITA4 & SEGUNDA & 20 & 141 & 992 \\
\hline ESQUISTO & ESOUISTO7 & SEGUNDA & 17 & 90 & 986 \\
\hline CALIZA & CALIZA5 & SEGUNDA & 11 & 63 & 1144 \\
\hline BASALTO 2 & RICHINAL1 & SEGUNDA & 20 & 134 & 1000 \\
\hline OFITA & OFITA1 & SEGUNDA & 6,5 & 45 & 854 \\
\hline
\end{tabular}


La absorción se destacó como la propiedad fundamental del árido que mayor influencia produjo en la consistencia del hormigón. Si observamos los resultados de absorción para los distintos áridos, los valores mayores se obtuvieron con el basalto 1 y la caliza, lo cual se reflejó posteriormente en las consistencias obtenidas. Mientras para igualdad de condiciones de la mezcla el pórfido permitió obtener consistencias blandas y fluidas la caliza producía mezclas de consistencia plástica y el basalto de consistencia seca.

La importancia de controlar la absorción del árido queda reflejada por estos resultados, ya que ello influirá directamente en la consistencia. Sin embargo, teniendo en cuenta que las operaciones de amasado y llenado de las probetas eraun proceso que podía durar aproximadamente quince o veinte minutos, surgió la duda de cuánta agua podían absorber los áridos durante este período. En la Fig. 5 se ve la evolución del coeficiente de absorción desde el tiempo de 1 minuto al de 24 horas, para un árido de baja absorción (el esquisto) y de absorción media (la caliza). Se observa que, al menos para estos valores de absorción, no existen grandes diferencias entre los valores obtenidos a 1 minuto y a 1 día, lo cual nos indica que la absorción es un fenómeno que se desarrolla muy rápidamente. Los áridos absorben el agua directamente durante el proceso de amasado en la hormigonera, restándosela a la masa y por tanto disminuyendo la trabajabilidad. Hay que tener en cuenta que dado el elevado peso de árido grueso que lleva la amasada, el contenido de agua que se resta a la masa puede llegar a ser muy elevado. Por otra parte se observa una cierta variabilidad en los resultados de absorción medida en diferentes tiempos, lo cual es debido en parte a la subjetividad que conlleva el ensayo en sí: Antes de proceder a la pesada, hay que secar el árido (previamente sumergido en agua) con un paño para que quede húmedo con la superficie seca.

Si los áridos tienen un nivel de absorción similar, en la consistencia de la amasada entrarán a influir otros factores, ya secundarios, tales como el coeficiente de forma o el tamaño máximo de los mismos.

En el caso de caliza y basalto 1 influye en la consistencia el hecho de que tengan un tamaño máximo algo inferior al resto de los áridos, lo cual implica una mayor superficie a mojar por la pasta. El tamaño máximo se calculó de acuerdo al método de Faury. El esquisto presentó el coeficiente de forma menor, lo cual también pudo influir en originar menor trabajabilidad.

El árido que proporcionó mejores consistencias fue la monzonita, con valores bajos de absorción y un buen coeficiente de forma y además una granulometría que permitió, con la arena que se utilizaba, obtener curvas granulométricas idénticas a la de referencia propuesta por Faury. En las Figs. 6a y $6 \mathrm{~b}$ se muestran las granulometrías de las dosificaciones "HSC18P" y "MONZONITA4". Se puede observar el perfecto ajuste entre curvas de referencia y composición en el caso de la monzonita, sensiblemente peor en el caso del pórfido. Esto, junto con un mejor coeficiente de forma, permitió mejorar aun más las consistencias conseguidas con el árido porfídico.

En cuanto a las resistencias obtenidas con los distintos áridos, éstas quedan reflejadas en el Cuadro 5. Pórfido, monzonita y esquisto proporcionaron similares resistencias. Se trata de áridos de similares características mecánicas reflejadas por su coeficiente de Los Angeles y su índice de machacabilidad (Cuadro 4). Este último ensayo refleja mejor la resistencia estática del árido, esfuerzo al que va a estar sometida la probeta en el ensayo de compresión, mientras que el coeficiente de Los Angeles nos indica una buena resistencia al desgaste. Ambas resistencias normalmente están directamente relacionadas, pero no siempre es así, como sucede en el caso del pórfido, con una resistencia al desgaste no tan buena como la que presentaron otros áridos con similar índice de machacabilidad.

La caliza y el basalto 1 proporcionaron las máximas resistencias. Ambos presentaron coeficientes de absorción también superiores a los áridos antes citados, lo cual como ya se ha comentado se reflejó en la consistencia. Sin embargo, desde el punto de vista de la resistencia, este fenómeno es bueno ya que resta agua a la mezcla, que es absorbida por el árido y queda luego disponible para prolongar el curado del hormigón. La relación agua/ cemento efectiva disminuye y además se mejoran las condiciones del curado.

El resultado obtenido con el árido calizo resulta sorprendente si se compara con la ofita. Ambosáridos eran débiles, porosos y absorbentes, pero la caliza produjo las resistencias más altasy la ofita las más bajas. La explicación pudiera estar en la adherencia epitáxica pasta-árido que se desarrollaen general con losáridos calizos. Esta adherencia queda reflejada en la Fotografia 5, de un trozo de probeta rota, donde se observa claramente la fisura que avanza partiendo limpiamente los áridos que encuentra a su paso. Esto nos lleva a pensar que las resistencias conseguidas con este árido aun pueden ser ampliamente superadas utilizando calizas densas de buena calidad, que presenten un bajo coeficiente de Los Angeles y un bajo índice de machacabilidad, sumando el efecto de utilizar un árido resistente al de que además presente unabuena adherencia a la pasta.

Por tanto, las recomendaciones finales para la selección del árido grueso para la fabricación de hormigón de alta resistencia, se pueden resumir como sigue:

.Como factor principal de cara a la consistencia, limitar al máximo la absorción del árido. Se recomienda utilizar áridos con absorción próxima al $1 \%$. 
Controlar el coeficiente de forma del árido, utilizando siempre que sea posible áridos con coeficientes próximos o superiores a 0,25 .

En cuanto a consistencia se refiere, tamaños máximos de $12-14 \mathrm{~mm}$ son mejores que los de $10 \mathrm{~mm}$, ya que presentan menor superficie específica y demandarán menos agua.

Utilizar granulometrías cuidadas, de tal manera que se logre un buen ajuste a las curvas de referencia. En nuestro caso la curva de referencia utilizada fue la que propone el método de dosificación de Faury.

.Desde el punto de vista de resistencia interesa utilizar áridos con un coeficiente de los Angeles próximo a 15 y un índice de machacabilidad próximo a 13, aunque ciertos áridos calizos puedan compensar una debilidad del material con una adherencia al mortero de cemento excelente.
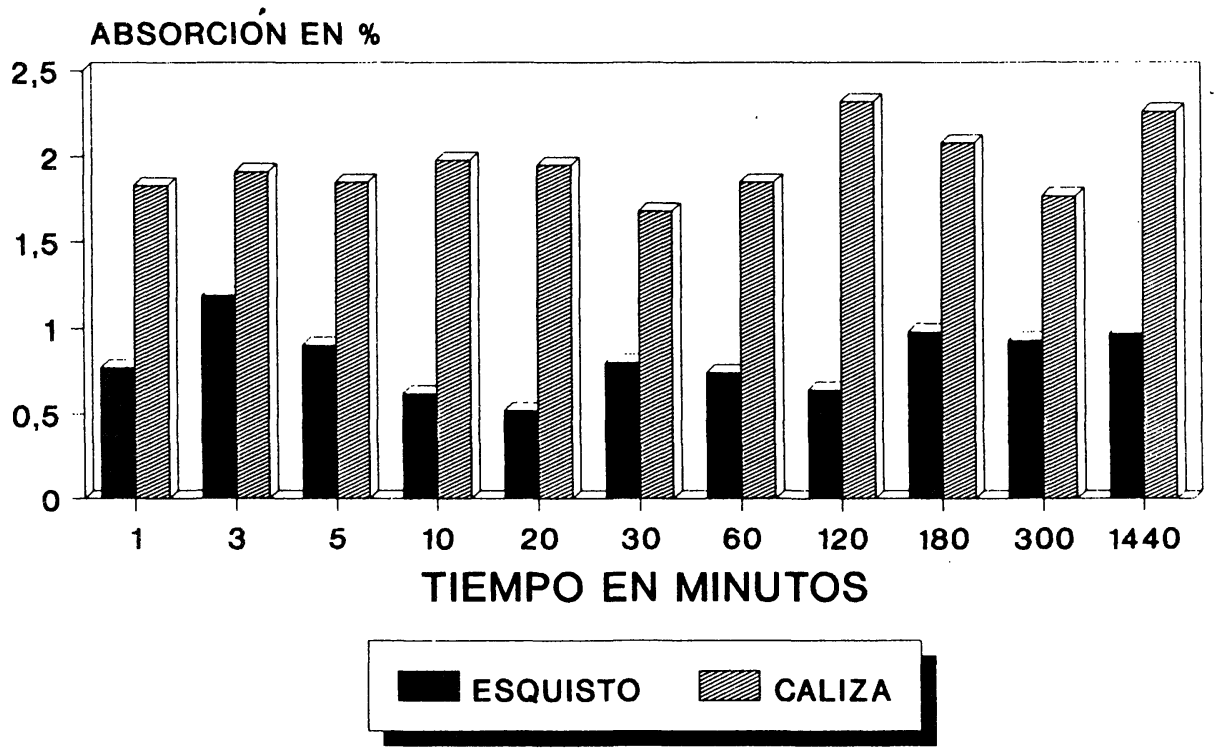

Fig.5.- Evolución de la absorción en el tiempo.

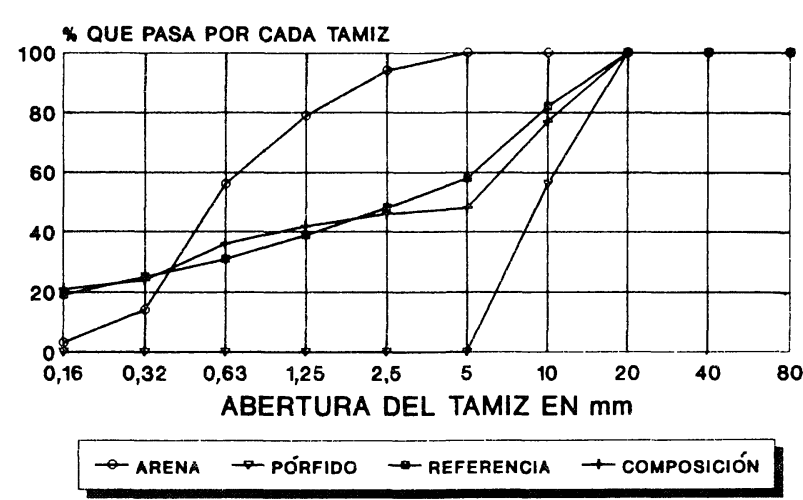

Fig. 6a.- Granulometrias dosificación pórfido (HSC 18P)

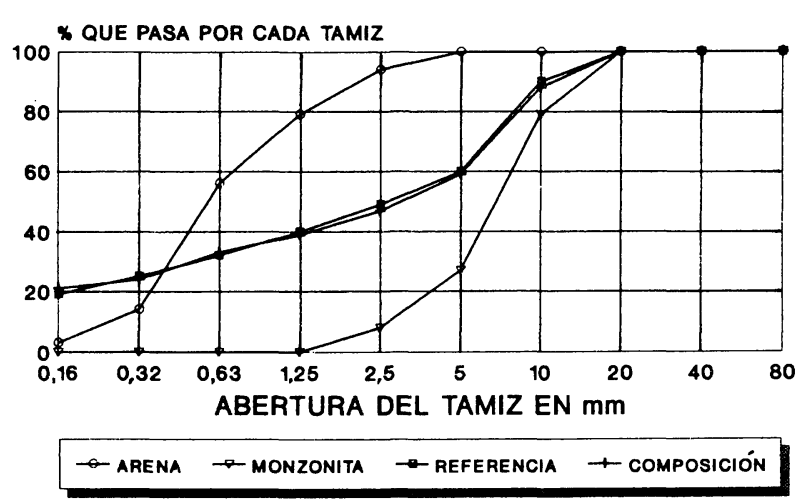

Fig. 6b.- Granulometrías dosificación monzonita 4

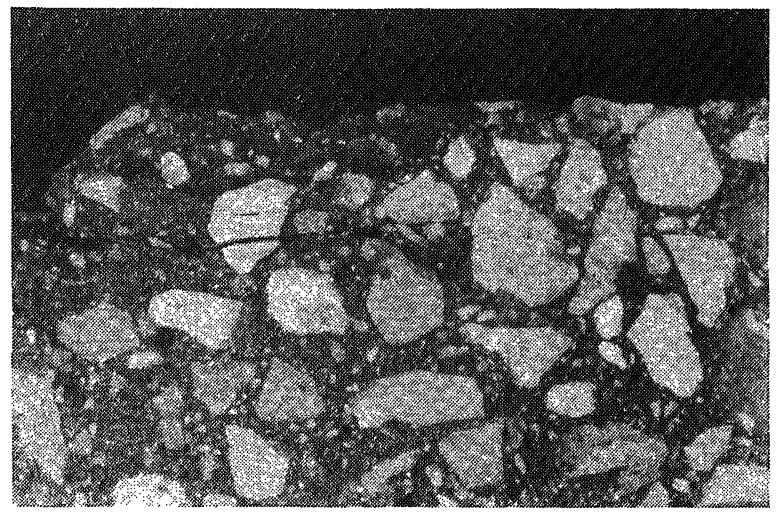

Foto 5 


\section{3.- CONCLUSIONES}

Una buena selección de materiales para fabricar hormigones de alta resistencia es la principal garantía de éxito, sobre todo cuando se desea agotar las posibilidades para llegar a los niveles más elevados de resistencia.

.El incremento de resistencia en hormigones con cemento I55-A en comparación a hormigones con cemento I45-A disminuye al aumentar la resistencia del hormigón. En hormigón de alta resistencia el incremento estará alrededor del 3-4\%. Sin embargo, el hormigón con cemento I55-A presentará en general más problemas de consistencia (ya que normalmente la mayor categoría resistente se consigue gracias a una mayor finura de molido), pudiendo no alcanzarse relaciones agua/cemento muy bajas con consistencia adecuada. Predeterminar la selección del cemento a los de máxima categoría resistente puede resultar un error, sobre todo si se quiere bajar mucho la relación agua/cemento, a no ser que el cemento sea de mayor resistencia por tener una composición química más ajustada.

Otro factor importante que se debe controlar en el cemento, es su fraguado, que si resulta excesivamente lento puede presentar problemas con elevadas cantidades de superplastificante, a veces necesarias para los niveles más altos de resistencia.

Elegir un binomio adecuado cemento-superplastificante es básico para llegar a determinados niveles de relación agua/cemento. De acuerdo a los resultados obtenidos en el programa de ensayos, se da una metodología de selección de cementos de baja demanda de agua, y aditivos efectivos con dichos cementos.

.El humode sílice debe elegirse de acuerdo al cumplimiento de la normativa vigente. El paso siguiente es exigir el máximo contenido de $\mathrm{SiO}_{2}$ activo para conseguir una elevada actividad puzolánica, que se reflejará en mayores incrementos de la resistencia.

.Una vez agotada la vía de reducir al máximo la relación agua/cemento(cuyo límite lo marcará el binomio cementosuperplastificante, la demanda de agua de cemento y áridos y la consistencia que se quiere conseguir), para incrementar más la resistencia se debe concentrar el esfuerzo en la selección cuidada del árido grueso. De acuerdo a los resultados experimentales, se recomienda la selección del árido según el resultado en ensayos como el coeficiente de Los Angeles, índice de machacabilidad, coeficiente de absorción, coeficiente de forma y granulometría.

\section{BIBLIOGRAFÍA}

(1) ARREDONDO, F. (1965)

Dosificación de hormigones. Manuales y normas del Instituto Eduardo Torroja de la Construcción y del Cemento, 1965.

(2) BLICK, R.L.; PETERSEN, C.F.; WINTER, M.E. (1974) "Proportioning and controlling High Strength Concrete". ACI SP-46, Proportioning concrete mixes, Editor: ACI Committee 211, 1974, pp. 141-156.

(3) CARRASQUILLO,P.M.; CARRASQUILLO, R.L. (1988)

"Evaluation of the use of current concrete practice in the production of high-strength concrete". ACI Materials Journal, January-February 1988, pp. 49-54.

(4) CARRIL CARVAJAL, C.(1987) “Hormigones de alta resistencia”. Revista O. Públicas, Sept. 1987, pp. 543-553.

(5) COLLEPARDI, M.; MONOSI, S.; VALENTE, M. (1989)

"Optimization of SP type and dosage in FA and SF concretes". ACI SP-119, Superplasticizers and other chemical in concrete, Editor: V.M. Malhotra, 1989, pp. 425-443.

(6) COLLINS, T.M. (1989)

"Proportioning High Strength Concrete to control creep and shrinkage". ACI Materials Journal, Nov-Dec 1989, pp. 576-580.

(7) DETWILER, R.J.; MEHTA, P.K. (1989)

"Chemical and physical effects of silica fume on the mechanical behaviour of concrete". ACI Materials Journal, Nov-Dec. 1989, pp. 609-614.

(8) EDDINE, J.; CALAVERA, J.; FERNANDEZ, J. (1992)

"Proyecto de dosificación de un hormigón de alta resistencia". Congreso Intercontinental del Hormigón Preparado, Madrid, Junio 1992. Parte VI/A. 
(9) FERRARIS, CH.F.; GAIDIS, J.M. (1992)

"Connection between the rheology of concrete and rheology of cement paste". ACI Materials Journal, July-August 1992, pp. 388-393.

(10) HANNA, E.; LUKE, K.; PERRATON, D.; AITCIN, P.C. (1989)

"Rheological behaviour of portland cement in the presence of a superplasticizer". Proceeding Third International Conference Ottawa, Canada, 1989, ACI SP-119, Superplasticizers and other chemical in concrete, Editor: V.M. Malhotra, pp. 171-188.

(11) HELLAND, S. (1986)

"Silica fume in High Strength Concrete". Nordisk Betong, Jan-Feb. 1986, pp.91-95.

(12) HERRERO NUÑEZ, E. (1992)

“Aridos para hormigones de altas resistencias, sus características". Cemento-Hormigón, №709, Agosto 1992, pp.1136-1142.

(13) KAPLAN, M.F. (1958)

"The effects of the properties of coarse aggregates on the workability of concrete". Magazine of Concrete Research, August 1958, pp.63-74.

(14) LARRARD, F. (1990)

"A method for proportioning High Strength Concrete". Cement, Concrete and Aggregates, V. 12, №2, Summer 1990, pp. 4752.

(15) LARRARD, F. (1992)

"Relation entre formulation et quelques propietes mecaniques des betons a hautes performances". Materials and Structures, $N^{\circ}$ 45, 1992, pp. 464-475.

(16) LARRARD, F.; ACKER, P.; MALIER, Y. (1987)

"Very High Strength Concrete from the laboratory to the construction site". Utilization of High Strength Concrete, Proceedings of the Symposium in Stavanger, Norway. Editores:I.Holand, B.Jakobsen, S.Helland y R.Lenschow, 1987, pp. 509-516.

(17) LARRARD, F.; GORSE, J.F.; PUCH, C. (1990)

"Efficacités comparées de diverses fumeés de silice comme additif dans les bétons à hautes performances". Bulletin du Liaisons Lab. P. et. Ch., $\mathrm{N}^{\circ} 168$, Juillet-Aout 1990, pp. 97-105.

(18) MEHTA, P.K.; AITCIN, P.C. (1990)

"Microstructural basis of selection of materials and mix proportions for High Strength Concrete". H.S.C. Second International Symposium on utilization of high strength concrete (Berkeley, California) ACI SP-121, Editor W.T. Hester, 1990, pp. 265-286.

(19) NEVILLE, A.M. (1981)

Properties of concrete. Editor: Longman Scientific \& Technical. 1981

(20) OLIVIER, M.; AGUADO, A. (1992)

"Hormigones de alta resistencia. Una propuesta de definición y de clasificación". Congreso Intercontinental del Hormigón Preparado, Madrid, Junio 1992. Parte V/A.

(21) PCI COMMITTEE ON HRWRA (1981)

"Recommended practice for use of HRWRA in precast prestressed concrete operations". PCI Journal, Sept-Oct. 1981, pp. 2849.

(22) PENTTALA, V. (1986)

"Compatibility of binder and superplasticizer in High Strength Concrete". Nordic Concrete Research Publication, N" 5, 1986, pp. 117-128.

(23) RAMACHANDRAN, V.S.; BEAUDOIN, J.J.; SHIHUA, Z. (1989)

"Control of slump loss in SP concrete". Materials and Structures, March 1989, Vol. 22, № 128, pp. 107-111.

(24) YAMAMOTO, Y.; KOBAYASHI, M. (1982)

"Use of mineral fines in High Strength Concrete- water requirement and strength". Concrete International, July 1982, pp. 3340. 